(1)

CrossMark

\title{
New Xpert MTB/XDR: added value and future in the field
}

\author{
Arnold Bainomugisa ${ }^{1}$, Christopher Gilpin ${ }^{2}$, Christopher Coulter ${ }^{1}$ and \\ Ben J. Marais ${ }^{3}$
}

Affiliations: ${ }^{1}$ Queensland Mycobacterium Reference Laboratory, Pathology Queensland, Brisbane, Australia. ${ }^{2}$ International Organization for Migration, Geneva, Switzerland. ${ }^{3}$ The Marie Bashir Institute for Infectious Diseases and Biosecurity (MBI), University of Sydney, Sydney, Australia.

Correspondence: Arnold Bainomugisa, Queensland Mycobacterium Reference Laboratory, Pathology Queensland, Royal Brisbane and Women Hospital complex, Block 7, Level 5, Brisbane, Australia. E-mail: Arnold.bainomugisaßahealth.qld.gov.au

@ERSpublications

Xpert MTB/XDR is a new rapid molecular test for drug resistant tuberculosis https://bit.ly/3l2t4op

Cite this article as: Bainomugisa A, Gilpin C, Coulter C, et al. New Xpert MTB/XDR: added value and future in the field. Eur Respir J 2020; 56: 2003616 [https://doi.org/10.1183/13993003.03616-2020].

\section{Introduction}

The recent launch of the new Xpert MTB/XDR assay (Cepheid Inc., Sunnyvale, CA, USA) by the Foundation for Innovative New Diagnostics [1] was widely welcomed by the tuberculosis (TB) community, given the critical need for new and better diagnostics to guide the treatment of drug resistant tuberculosis (DR-TB). Xpert MTB/XDR detects resistance to isoniazid (target genes: inhA promoter, katG, fabG1, oxyR-aphC intergenic region), ethionamide (inhA promoter), fluoroquinolones ( $g y r A$ and $g y r B$ ), and second-line injectables ( $r r s$ and eis promoter) and is positioned as an add-on or "reflex test" in patients with rifampicin resistance detected by Xpert MTB/RIF or Ultra [2, 3].

The spread of DR-TB strains threatens recent gains in global TB control [4], with evidence that the majority of patients with rifampicin resistant (RR-TB) or multi-drug resistant (MDR-TB) TB acquire their infection through person-to-person transmission. Inadequate diagnostic and treatment options have hampered an effective global response. The use of Xpert MTB/RIF as a rapid and sensitive frontline TB detection test has been shown to improve patient outcomes and is cost effective [5], but data for RR/ MDR-TB are lacking; partly hampered by the poor treatment options available in the past. We provide a brief overview of the perceived benefits, limitations and remaining challenges with the new test (table 1).

\section{Benefits/added value}

Existing Xpert MTB/RIF and Ultra assays only detect rifampicin resistance, which prevents RR/MDR-TB differentiation and provides no further treatment guidance. Expanded drug susceptibility testing (DST) is required to guide optimised RR/MDR-TB treatment, which is essential to improve patient outcome, reduce the duration of infectiousness and limit the risk of drug resistance amplification. Drug resistance amplification is a particular concern with the promotion of new bedaquiline containing all-oral short-course regimens [6]. Culture and phenotypic DST is the current gold standard, but is rarely available in TB endemic settings. In the absence of phenotypic DST, molecular techniques such as line probe assays (e.g. MTBDRsl) are recommended. However, these assays have poor sensitivity in sputum smear negative patients and require specialised infrastructure that is not available outside well-equipped central laboratories [7]. 
TABLE 1 Overview of perceived benefits and limitations of the new Xpert MTB/XDR assay

Benefits/added value

\section{Rapid extended drug resistance profiling}

The slow speed of traditional phenotypic testing precludes early initiation of optimised therapy, compromising patient outcomes and amplifying drug resistance

\section{Limited infrastructure requirements}

Rapid expanded genotypic testing can be performed using the robust GeneXpert platform, with proven track record in the field and TB programme familiarity

\section{Detection of fluoroquinolone resistance}

This is essential to optimise second-line treatment regimens; especially to protect bedaquiline against emerging resistance in all-oral short-course regimens

\section{Detection of isoniazid resistance}

Key precursor of MDR strains and need for optimised treatment to reduce disease relapse and drug resistance amplification with standard first-line treatment

\section{Detection of ethionamide resistance}

Useful to know if ethionamide may still be a useful agent, information on inhA promotor mutations could also guide high dose isoniazid therapy

\section{Resistance to second-line injectables}

Important to know when newer all-oral RR/MDR TB regimens are unavailable, or if the patient cannot be adequately treated by all-oral regimens

\section{Limitations/challenges}

Genotypic drug resistance testing fails to detect all phenotypic drug resistance; risk of diagnostic selection of undetected mutations as observed with Xpert MTB/RIF

Uncertainty about its performance in patients with pauci-bacillary disease and in Xpert Ultra "trace positive" or "intermediate resistance" patients

Need for additional performance assessment in different geographical settings and with a variety of clinical specimens; uncertain sensitivity with extra-pulmonary and pauci-bacillary specimens

Detects mutations only in "hot spots" of target genes; caution regarding potential discrepancy between geno- and phenotypic drug resistance profiles

Minor discrepancy between geno- and phenotypic drug resistance profiles; relatively high cost for MDR-TB testing as a reflex test of Xpert MTB/RIF or Ultra

Will not detect non-MDR isoniazid resistance if only used as a follow-on test when Xpert MTB/RIF or Ultra is positive

More common discrepancy between geno- and phenotypic drug resistance profiles; limited phenotypic DST availability in most settings

Detection of second-line injectables less relevant since new all-oral regimens are preferred; amikacin identified as the preferred injectable and now regarded as a WHO category C (less important) drug for RR/MDR-TB treatment

No resistance detection for new or re-purposed agents

TB: tuberculosis; RR: rifampicin resistance; MDR: multidrug resistant (resistance against isoniazid and rifampicin); DST: drug susceptibility testing.

A key benefit of the Xpert MTB/XDR assay is that it requires the same sample processing steps as Xpert MTB/RIF and Ultra, which have demonstrated robustness in field conditions. The familiarity, portability and user-friendly format of the Xpert MTB/XDR assay makes it attractive for decentralisation. It also has minimal biosafety requirements, offers rapid turn-around times and can be operated by health personnel with limited training. Results are available in less than $90 \mathrm{~min}$, compared to several weeks with traditional culture-based DST or several days with line probe assays performed at a central laboratory level.

Rapid fluoroquinolone resistance determination is critical, given its pivotal role in RR/MDR-TB treatment and importance in protecting companion second-line drugs like bedaquiline [8, 9]. The 2020 World Health Organization (WHO) recommendations for the treatment of RR/MDR-TB recognise the key contribution of later generation fluoroquinolones in shorter duration all-oral regimens [6]. In initial evaluations, Xpert MTB/ XDR was able to identify fluoroquinolone resistance with $91.4 \%$ sensitivity and $98.5 \%$ specificity compared to phenotypic DST [3]. This is close to WHO targets for diagnostic sensitivity (>95\%) and specificity (>98\%) of rapid DST [10]. However, the assays' diminished ability to detect mutations (S91P/A90V, D94A) causing low level fluoroquinolone resistance, especially in hetero-resistant strain populations [3], is a concern and highlights the need for further careful monitoring of discrepant geno/phenotypic DST results in different settings.

\section{Limitations/challenges}

Apart from cartridge costs, the new assay requires GeneXpert instrument modules with 10 colour multiplex technology, hence the current Xpert instruments will require major refitting and recalibration. This presents a significant challenge, particularly to low- and middle-income countries that may need to shoulder additional shipping and servicing expenses. Although the assay is attractive for decentralisation, 
its optimal placement in different settings needs to be guided by local diagnostic algorithms, as well as local feasibility and cost-effectiveness. Poor infrastructure, cost and logistical constraints will limit point-of-care placement in most high burden countries [5]. Another imperative to consider is the ability to provide adequate RR/MDR-TB treatment in all settings with diagnostic access [11]. In recent years, MDR-TB programme up-scaling and increased access to high quality second-line TB drugs through the Global Drug Facility successfully narrowed the RR/MDR-TB diagnosis-treatment gap resulting from Xpert MTB/RIF roll-out.

Isoniazid resistance is a key precursor to $\operatorname{MDR}-\mathrm{TB}[12,13]$, which indicates the need for improved detection and appropriate treatment of non-MDR isoniazid resistant strains that are not detected by Xpert MTB/RIF or Ultra. Few countries have laboratory capacity to assess isoniazid resistance [14], which the new assay has excellent capacity to detect; sensitivity $98.3 \%$ and specificity $95 \%$ compared to phenotypic DST [3]. This is important given that WHO recommends a modified treatment regimen for these patients to improve treatment outcomes and to limit MDR-TB generation [6]. However, with placement as a "reflex test" this ability will not be utilised and it would have been of greater value if isoniazid and rifampicin resistance testing was combined in a frontline diagnostic. It should be noted that isoniazid resistance (including high-level resistance) can be conferred by mutations in genes not targeted by the Xpert MTB/XDR, such as $a p h C, n d h, m s h A$ and $m y m A$. These mutations are uncommon, but their geographic distribution has not been assessed. Furthermore, their frequency may increase with diagnostic selection, a phenomena observed in Swaziland where rifampicin resistance mediated by mutation outside the resistance hotspot has become common [15].

Ethionamide has chemical similarity to isoniazid and shares a final common pathway targeting mycolic acid biosynthesis. Ethionamide resistance is evaluated through identification of mutations in the inhA promoter region known to confer co-resistance to isoniazid (low level) and ethionamide (high level) [16, 17]. However, there are additional genes (ethA, ethR, $n d h$ and $m s h A)$ and unknown mechanisms that also confer resistance [18]. Since phenotypic ethionamide DST is lacking in most settings [19], genotypic approaches are valuable for identification of ethionamide resistance. Although new WHO guidelines classify ethionamide as a group C drug that is only considered when newer/re-purposed agents are not available [6], it remains a potent agent to consider and knowledge of $i n h A$ promoter mutations also guide potential use of high dose isoniazid [20].

Given evidence for improved treatment outcomes and reduced adverse effects, new RR/MDR-TB treatment guidelines prioritise the use of oral agents (e.g. bedaquiline, linezolid, clofazimine, cycloserine) $[6,21]$. Therefore, the detection of resistance to injectable agents has become less relevant. However, knowledge of susceptibility to injectable drugs remains useful in instances where an effective all-oral treatment regimen cannot be assembled and if adequate measures to monitor adverse effects are in place [22]. With positioning as a "reflex test", minimum sample volume to permit follow-on testing and conditions for sample storage will have to be critically evaluated to streamline logistics and maintain good performance.

\section{Future in the field}

As with the implementation of Xpert MTB/RIF and Ultra, future studies should assess the performance of Xpert $\mathrm{MTB} / \mathrm{XDR}$ in different geographical settings and with different clinical specimens. This is particularly important in people living with HIV and in children, in whom extra-pulmonary and paucibacillary disease are more common [23, 24]. Samples such as fine needle aspiration biopsies may deliver excellent yields, as for Xpert MTB/RIF [25], while reduced sensitivity is expected in cerebrospinal fluid, although a positive test will have major clinical significance [26]. The new assay demonstrated comparable sensitivity to Xpert MTB/RIF in pulmonary samples, but its performance using extra-pulmonary samples has not been evaluated [3, 27]. The limit of detection for Mycobacterium tuberculosis by Xpert MTB/XDR $\left(71.9 \mathrm{CFU} \cdot \mathrm{mL}^{-1}\right)$ is comparable to MTB/RIF $\left(86.9 \mathrm{CFU} \cdot \mathrm{mL}^{-1}\right)$, but not as low as Ultra (15.6 CFU.mL $\left.{ }^{-1}\right)$. How to deal with Xpert Ultra "trace" calls will have to be discussed by an independent WHO-convened guideline development group, who will consider different scenarios for incorporating the new assay into existing diagnostic algorithms.

As WHO looks to expand the capacity for detection of DR-TB, new rapid molecular diagnostic technologies are critical to improve TB control and progress towards ultimate TB elimination [28]. The new assay will improve rapid diagnosis of patients with second-line drug resistance, enabling early initiation of appropriate treatment and optimal person-centred care. However, the assay only targets a limited number of resistance variants recognised as "hot spots" in particular target genes, which may lead to variable sensitivity in different settings. The detection of isoniazid resistance should ideally be included with a front-line TB detection test, not as an add-on after rifampicin resistance has been detected. The main value of the assay is the rapid detection of fluoroquinolone resistance to guide all-oral MDR-TB treatment, but a caveat is the fact that it does not detect resistance against newer/re-purposed drugs 
included in the regimen. Routine whole genome sequencing (WGS) overcomes this shortcoming by interrogating the entire genetic repertoire, but this is currently only feasible in high resource settings and a full understanding of the genetic determinants of resistance against newer/re-purposed drugs is lacking. Moreover, in most instances WGS still requires initial culture of the specimen, which introduces potential strain biases and critical delays in patient management.

A key benefit of the Xpert MTB/XDR assay is the robustness of the Xpert platform, its minimal biosafety requirements and the fact that TB programmes are already familiar with its use. Its launch is timely, given the urgent need for rapid fluoroquinolone resistance determination to guide the use of new all-oral short-course RR/MDR-TB regimens, and especially to protect bedaquiline against the rapid emergence of drug resistance. However, strategies that integrate the new assay into existing algorithms require careful consideration of how to extract optimal value and establish appropriate monitoring processes in a variety of settings. While molecular targets chosen by the manufacturers several years prior may no longer be as contemporary as desired, this new assay successfully demonstrates rapid multiplex technology suitable for near patient testing that is potentially amenable to inclusion of alternate target mutations.

Conflict of interest: None declared.

\section{References}

1 FIND. New Rapid Molecular Test for Tuberculosis can Simultaneously Detect Resistance to First- and Second-line Drugs. www.finddx.org/newsroom/pr-16jul20/ Date last updated: 16 July 2020. Date last accessed: 16 July 2020.

2 Chakravorty S, Roh SS, Glass J, et al. Detection of isoniazid-, fluoroquinolone-, amikacin-, and kanamycin-resistant tuberculosis in an automated, multiplexed 10-color assay suitable for point-of-care use. J Clin Microbiol 2016; 55: 183-198.

3 Cao Y, Parmarl H, Gaur R, et al. Xpert MTB/XDR: a ten-color reflex assay suitable for point of care settings to detect isoniazid, fluoroquinolone, and second line injectable drug-resistance directly from Mycobacterium tuberculosis positive sputum. BioRxiv 2020; preprint [https://doi.org/10.1101/2020.09.08.288787].

4 Abubakar I, Zignol M, Falzon D, et al. Drug-resistant tuberculosis: time for visionary political leadership. Lancet Infect Dis 2013; 13: 529-539.

5 Pooran A, Theron G, Zijenah L, et al. Point of care Xpert MTB/RIF versus smear microscopy for tuberculosis diagnosis in southern African primary care clinics: a multicentre economic evaluation. Lancet Glob Health 2019; 7: e798-e807.

6 World Health Organization. WHO Consolidated Guidelines on Drug-resistant Tuberculosis Treatment. www.who. int/tb/publications/2019/consolidated-guidelines-drug-resistant-TB-treatment/en/ Last updated: December 2019. Date last accessed: 28 June 2020.

7 Tomasicchio M, Theron G, Pietersen E, et al. The diagnostic accuracy of the MTBDRplus and MTBDRsl assays for drug-resistant TB detection when performed on sputum and culture isolates. Sci Rep 2016; 6: 17850.

8 Migliori GB, Lange C, Girardi E, et al. Fluoroquinolones: are they essential to treat multidrug-resistant tuberculosis? Eur Respir J 2008; 31: 904-905.

9 Tweed CD, Dawson R, Burger DA, et al. Bedaquiline, moxifloxacin, pretomanid, and pyrazinamide during the first 8 weeks of treatment of patients with drug-susceptible or drug-resistant pulmonary tuberculosis: a multicentre, open-label, partially randomised, phase 2b trial. Lancet Respir Med 2019; 7: 1048-1058.

10 World Health Organization. High-priority Target Product Profiles for New Tuberculosis Diagnostics: Report of a Consensus Meeting. www.who.int/tb/publications/tpp_report/en/ Last updated: December 2014. Date last accessed: 4 Jan 2020

11 Heidi A, Ruvandhi RN, Chris I, et al. Development, roll-out and impact of Xpert MTB/RIF for tuberculosis: what lessons have we learnt and how can we do better? Eur Respir J 2016; 48: 516-525.

12 Cohen KA, Abeel T, Manson MA, et al. Evolution of extensively drug-resistant tuberculosis over four decades: whole genome sequencing and dating analysis of Mycobacterium tuberculosis isolates from KwaZulu-Natal. PLoS Med 2015; 12: e1001880.

13 Bainomugisa A, Lavu E, Hiashiri S, et al. Multi-clonal evolution of multi-drug-resistant/extensively drug-resistant Mycobacterium tuberculosis in a high-prevalence setting of Papua New Guinea for over three decades. Microb Genom 2018; 4: e000147.

14 Stagg HR, Lipman MC, McHugh TD, et al. Isoniazid-resistant tuberculosis: a cause for concern? Int J Tuberc Lung Dis 2017; 21: 129-139.

15 Sanchez-Padilla E, Merker M, Beckert P, et al. Detection of drug-resistant tuberculosis by xpert MTB/RIF in Swaziland. N Engl J Med 2015; 372: 1181-1182.

16 Miotto P, Tessema B, Tagliani E, et al. A standardised method for interpreting the association between mutations and phenotypic drug resistance in Mycobacterium tuberculosis. Eur Respir J 2017; 50: 1701354.

17 Sandgren A, Strong M, Muthukrishnan P, et al. Tuberculosis drug resistance mutation database. PLoS Med 2009; 6: e2.

18 Brossier F, Veziris N, Truffot-Pernot C, et al. Molecular investigation of resistance to the antituberculous drug ethionamide in multidrug-resistant clinical isolates of Mycobacterium tuberculosis. Antimicrob Agents Chemother 2011; 55: 355-360.

19 Van Ingen J, Simons S, De Zwaan R, et al. Comparative study on genotypic and phenotypic second-line drug resistance testing of Mycobacterium tuberculosis complex isolates. J Clin Microbiol 2010; 48: 2749-2753.

20 Dooley KE, Miyahara S, von Groote-Bidlingmaier F, et al. Early bactericidal activity of different isoniazid doses for drug-resistant tuberculosis (INHindsight): a randomized, open-label clinical trial. Am J Respir Crit Care Med 2020; 201: $1416-1424$. 
21 Gandhi NR, Brust JM, Sarita SN. A new era for treatment of drug-resistant tuberculosis. Eur Respir J 2018; 52 : 1801350

22 Nahid P, Mase SR, Migliori GB, et al. Treatment of drug-resistant tuberculosis an official ATS/CDC/ERS/IDSA clinical practice guideline. Am J Respir Crit Care Med 2019; 200: 93-142.

23 Dorman SE, Schumacher SG, Alland D, et al. Xpert MTB/RIF Ultra for detection of Mycobacterium tuberculosis and rifampicin resistance: a prospective multicentre diagnostic accuracy study. Lancet Infect Dis 2018; 18: 76-84.

24 Nicol MP, Workman L, Prins M, et al. Accuracy of Xpert Mtb/Rif Ultra for the diagnosis of pulmonary tuberculosis in children. Pediatr Infect Dis J 2018; 37: 261-263.

25 Ligthelm LJ, Nicol MP, Hoek KG, et al. Xpert MTB/RIF for rapid diagnosis of tuberculous lymphadenitis from fine-needle-aspiration biopsy specimens. J Clin Microbiol 2011; 49: 3967-3970.

26 Denkinger CM, Schumacher SG, Boehme CC, et al. Xpert MTB/RIF assay for the diagnosis of extrapulmonary tuberculosis: a systematic review and meta-analysis. Eur Respir J 2014; 44: 435-446.

27 Xie YL, Chakravorty S, Armstrong DT, et al. Evaluation of a rapid molecular drug-susceptibility test for tuberculosis. N Engl J Med 2017; 377: 1043-1054.

28 Lönnroth K, Migliori GB, Abubakar I, et al. Towards tuberculosis elimination: an action framework for low-incidence countries. Eur Respir J 2015; 45: 928-952. 